\title{
Cyclic AMP responsive element-binding protein promotes renal cell carcinoma proliferation probably via the expression of spindle and kinetochore-associated protein 2
}

\author{
Haihui Zhuang ${ }^{1,4}$, Xiangyu Meng ${ }^{1,4}$, Yanyuan Li $^{3}$, Xue Wang ${ }^{2,4}$, Shuaishuai Huang ${ }^{2,4}$, \\ Kaitai Liu ${ }^{5}$, Michael Hehir ${ }^{1,4}$, Rong Fang ${ }^{1}$, Lei Jiang ${ }^{1}$, Jeff X. Zhou ${ }^{1}$, Ping Wang ${ }^{1,4}$, \\ Yu Ren ${ }^{2,4}$ \\ ${ }^{1}$ Zhejiang Provincial Key Laboratory of Pathophysiology, Ningbo University School of Medicine, Ningbo 315211, China \\ ${ }^{2}$ Department of Urologic Surgery, Ningbo Urology and Nephrology Hospital, Ningbo 315000, China \\ ${ }^{3}$ Department of Pathology, First Affiliated Hospital, Zhejiang University School of Medicine, Hangzhou 310031, China \\ ${ }^{4}$ Laboratory of Kidney Carcinoma, Ningbo Urology and Nephrology Hospital, Urology and Nephrology Institute of Ningbo \\ University, Ningbo 315000, China \\ ${ }^{5}$ Ningbo Medical Center, LiHuiLi Hospital, Medical School, Ningbo University, Ningbo 315041, China \\ Correspondence to: Ping Wang, e-mail: pinoav@hotmail.com \\ Yu Ren, e-mail: nbrenyu@163.com
}

Keywords: $C R E B, S K A 2, R C C$, proliferation

Received: May 29, 2015

Accepted: January 01, 2016

Published: January 25, 2016

\section{ABSTRACT}

Emerging evidence shows that the aberrantly expressed cyclic AMP responsive element-binding protein (CREB) is associated with tumor development and progression in several cancers. Spindle and kinetochore-associated protein 2 (SKA2) is essential for regulating the progress of mitosis. In this study, we evaluate in vitro and in vivo the functional relationship between CREB and SKA2 in renal cell carcinoma (RCC). Suppressing and replenishing CREB levels were used to manipulate SKA2 expression, observing the effects on RCC cell lines. Computational prediction and ChIP assay identified that CREB targeted ska2 by binding its CRE sequence in the human genome. Overexpression of CREB reversed the inhibited cell growth following siSKA2 treatment, and reduced the number of cells holding in mitosis. Decreased expression of CREB suppressed RCC cell growth and xenograft tumor formation, accompanied by reduced expression of SKA2. In RCC tumor samples from patients, mRNA for SKA2 were plotted near those of CREB in each sample, with significantly increased immunohistochemical staining of higher SKA2 and CREB in the higher TNM stages. The study adds evidence that CREB, a tumor oncogene, promotes RCC proliferation. It probably achieves this by increasing SKA2 expression.

\section{INTRODUCTION}

Cyclic AMP responsive element-binding protein (CREB) is a proto-oncogenic transcription factor $[1,2]$, that generally regulates various cell functions by enhancing the expression of target genes [3, 4]. Aberrant expression of CREB has been well described in non-small cell lung carcinoma [5, 6], melanoma [7], breast [8-11], hepatocellular cancers [12] and acute myeloid leukemia $[13,14]$. Our previous reports showed that blocking the cyclic AMP-responsive element (CRE) site between CREB and targeted genes (Bcl-2 and cyclins), abrogated the anti-tumor drug-induced apoptosis and cell proliferation
$[15,16]$. It seemed that CREB played a pivotal role in promoting tumorigenesis. However, the role of CREB in renal cell carcinoma (RCC) remains less explored.

RCC ranks the third most frequent malignancy in urological oncology $[17,18]$. Like other cancers, the development of RCC is a multistep process with accumulation of genetic and downstream changes $[18,19]$. In order to find novel CREB-interacting proteins expressed in RCC cells, and to provide new insights into the cellular mechanisms, we identified a specific interaction between CREB and SKA2 (spindle and kinetochore-associated protein 2) by using bioinformatic software (http://mulan. dcode.org/) [20]. Although depletion of SKA2 can 
cause the cells to undergo a prolonged checkpoint in a metaphase-like state [21], little else is known about it.

The present studies showed that CREB is usually up-regulated in RCC tissues and cell lines. Decreased CREB expression significantly inhibited RCC cell proliferation in vitro and in vivo, accompanied by the suppression of SKA2 expression. Downregulation of SKA2 by RNAi significantly suppressed the proliferation of RCC cells and increased the cell number holding in mitosis, whereas these results could be reversed by the ectopic expression of CREB. Furthermore, analysis of clinical samples showed that the staining score of CREB was positively correlated with those of SKA2. These data suggest that CREB may function as a tumor oncogene to increase the cell proliferation by increasing expression of its target gene ska2 in RCC.

\section{RESULTS}

\section{CREB is frequently up-regulated in RCC}

CREB expression was initially evaluated in RCC tissues and matched adjacent non-tumor tissues. We assessed the protein expression of CREB levels by Western blot in 12 patient RCC samples, and found CREB was higher in tumor tissues than in non-tumor tissues in 9 patients (Figure 1A). Expression of CREB mRNA in RCC tissue was assessed by qRT-PCR in 40 patient RCC samples. The mRNA level of CREB was up-regulated in $30(75 \%)$ of tumor samples $(P<0.05)$ (Figure $1 \mathrm{~B})$. We used qRT-PCR to examine CREB expression in ACHN, 786-O and OS-RC-2 RCC cell lines and in normal proximal tubule epithelial cell line (HK-2). CREB expression was significant higher in $100 \%$ of RCC cells than HK-2 cells (Figure 1C). Furthermore, we found amplification of the CREB gene copy number in RCC cells compared with HK-2 (Figure 1D). These findings indicate that CREB was usually overexpressed in RCC tissues and cell lines, moreover the mechanism of the up-regulation of CREB is gene copy number amplification.

\section{Decreased expression of CREB suppresses RCC cell growth}

To explore the role of CREB in RCC cell proliferation, we down-regulated $\mathrm{CREB}$ expression to investigate the effect on cell proliferation in vitro. After transfecting specific CREB siRNA (339 and 486) into RCC cell lines and HK-2 cells, the mRNA and protein levels of CREB were significantly decreased, furthermore the efficacy of siCREB486 was greater than that of siCREB339 (Figure 2A, 2B). RCC cell proliferations were also significantly reduced, whereas, no effect was shown in HK-2 cells $(P<0.05$, Figure $2 \mathrm{C})$. We have previously reported that decreasing the pCREB inhibits the growth of RCC, by dominant negative CREB mutation in which the serine residue at 133 was replaced with threonine [22]. Here, we used two siRNA types to decrease the CREB expression and cause a significant inhibition of RCC cell proliferation. These results produce stronger evidence in vitro that suppression of CREB expression inhibits RCC cell proliferation.

\section{SKA2 is a CREB targeted gene}

To investigate the mechanism of CREB function in RCC carcinogenesis, we employed MultiTF tools (http:// mulan.dcode.org/) to look for putative human proteincoding gene targets of CREB. The gene ska2, which has the CRE site, was further studied as a potential target (Figure 3A). Down-regulation of CREB greatly reduced the mRNA and protein levels of SKA2 in ACHN, 786-O and OS-RC-2 cells (Figure 3B, 3C). ChIP assay also revealed that $\mathrm{CREB}$ bound to the promoter of SKA2 and increased protein expression in RCC cells (Figure 3D).

\section{SKA2 is involved in the CREB-regulated cell proliferation in vitro}

To explore whether SKA2 was involved in CREBregulated cell proliferation, we first explored the role of SKA2 in RCC growth, and found that SKA2 mRNA and protein levels in RCC cells were significantly higher than in HK-2 cells (Figure 4A). After successfully decreasing SKA2 mRNA levels (Figure 4B) by siSKA2 treatment, RCC cell proliferation was inhibited (Figure 4C). These data indicated that SKA2 could also independently influence tumor cell proliferation in vitro.

In addition, using pCI-neo/CREB vector containing the CREB coding sequence [23] increased the expression of CREB (Figure 4D) in OS-RC-2 RCC cells, to examine whether overexpression of CREB could counteract the reduction of SKA2 and the inhibition of cell proliferation. The result was that ectopic expression of CREB reversed the suppression of SKA2 mRNA (Figure 4E) and protein (Figure 4F), and reversed the decrease of cell proliferation (Figure 4G). In addition, siSKA2-treatment significantly increased the cell number holding in mitosis, whereas this was reversed by the combined treatment with siSKA2 and pCI-neo/CREB (Figure 4H).

Taken together, these results are consistent with our hypothesis that CREB probably promotes RCC cell proliferation by increasing SKA2 expression.

\section{Down-regulation of CREB suppresses xenograft tumor formation and reduces SKA2 expression in vivo}

To further evaluate the potential effect of CREB on RCC growth in vivo, OS-RC-2 cells were stablely transfected with scramble or shCREB by lentiviral plasmids and subcutaneously injected into nude mice. 

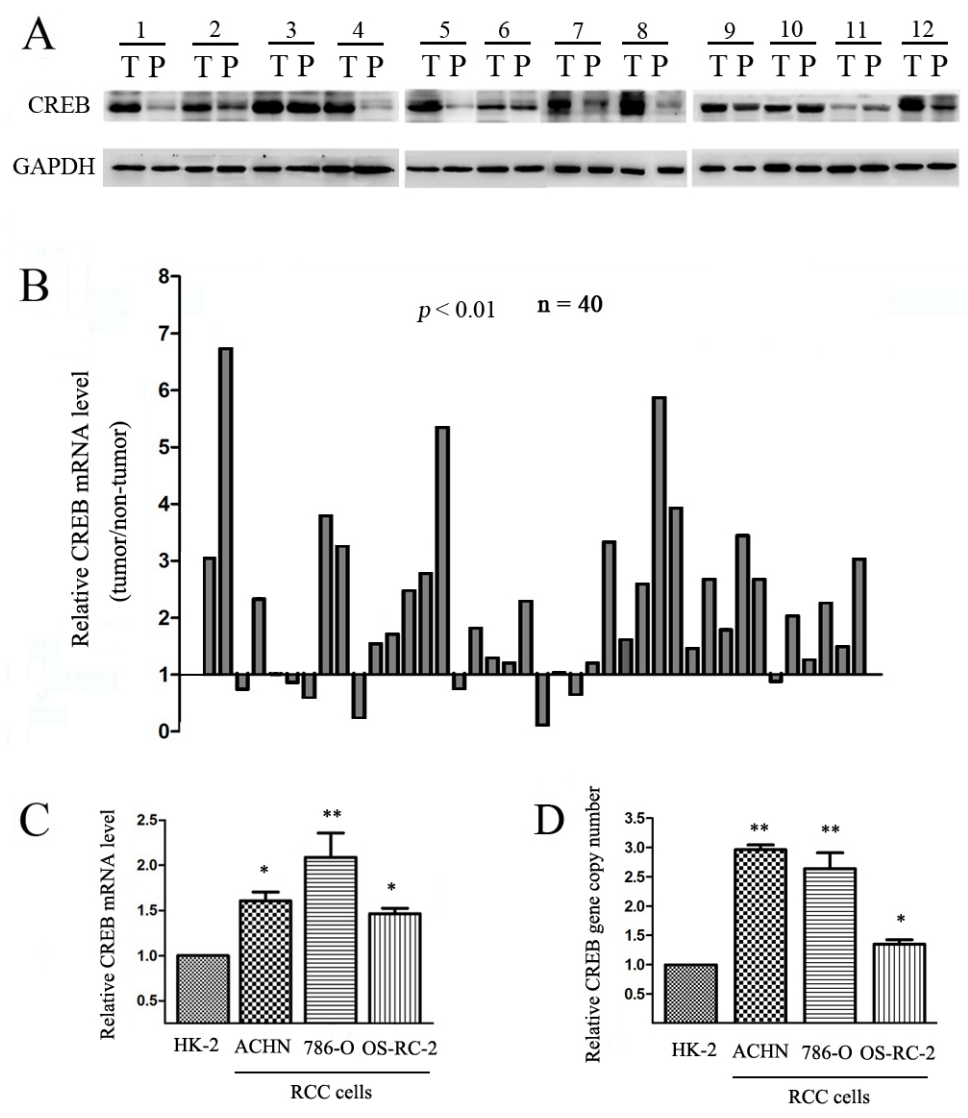

Figure 1: CREB expression is frequently up-regulated in RCC. Relative protein expression (A) and mRNA level (B) of CREB were detected in RCC tissues $(\mathrm{T})$ compared with their matched adjacent non-tumor tissues $(\mathrm{P})$. In three cell lines derived from RCC (ACHN, 786-O, OS-RC-2), CREB mRNA level (C) and gene copy number (D) were also higher than nonmalignant cell line (HK-2). Data were shown as mean $\pm \mathrm{SD}$ from three independent experiments. $* P<0.05, * * P<0.01$ versus HK-2 cell.
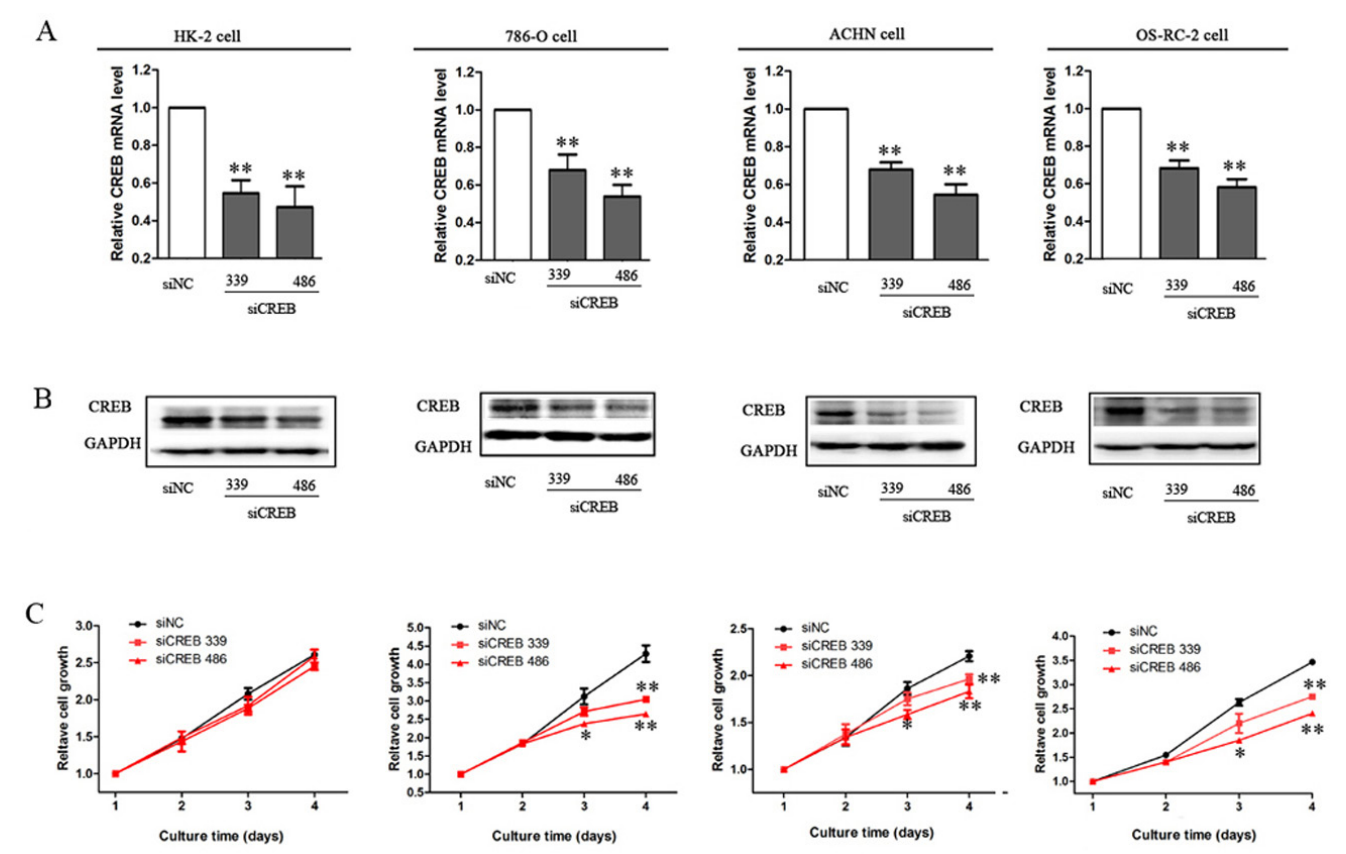

Figure 2: Decreased expression of CREB suppresses RCC cell growth. After cells were transfected with siCREB339 or siCREB486, the mRNA level (A) and protein expression (B) of CREB were significantly decreased in HK-2, ACHN, 786-O and OS-RC-2 cells, as compared with cells transfected with siNC. Whereas, inhibition of cell proliferation was not observed in HK-2 cell, but in RCC cells $(\mathbf{C})$. Data were shown as mean \pm SD from three independent experiments. ${ }^{*} P<0.05, * * P<0.01$ versus siNC. 
ShCREB treatment significantly decreased CREB expression levels in OS-RC-2 cells at both mRNA and protein levels (Figure 5A). 30 days after tumor cells transplantation, downregulated CREB led to a profound suppression of tumor growth, shown by a significant reduction in tumor volume and weight (Figure 5B, 5C). These data suggested that CREB inhibition decreased xenograft tumor formation of RCC cells in vivo.

To determine whether SKA2 was regulated by the effect of CREB in vivo, we tested SKA2 protein and mRNA levels in tumor tissues isolated from the mice which injected with OS-RC-2 cells containing shCREB or scramble. Weaker nuclear staining for SKA2 was shown in shCREB group than scramble group (Figure 5D). The distribution of staining scores for the tumor tissue was counted. In the scramble group, 4 mice had a score of $3+$ and 1 mice had a score of $1+$; meanwhile 2 mice had a score of $2+$ and 3 mice had a score of $1+$ in the shCREB group. Furthermore, Western blot analysis and
qRT-PCR indicated that decreasing levels of SKA2 was accompanied by CREB knockdown (Figure 5E, 5F). These data suggested that CREB knockdown can inhibition SKA2 expression in vivo.

\section{The association of CREB with SKA2 levels in RCC tissues}

Since we have shown that CREB was frequently increased in RCC and suggested targeting of SKA2 to increase cell proliferation, we investigated whether SKA2 expression level is associated with the malignancy in clinical samples. The results revealed that SKA2 mRNA level was significantly up-regulated in RCC tissues compared to their matched adjacent non-tumor tissues (Figure 6A). In serial sections of the same patient's tumor, the relative mRNA levels of SKA2 were plotted near that of CREB in each patient, a significant positive correlation was found ( $p<0.05 ; r=0.871$ ) (Figure 6B).

A

CRE
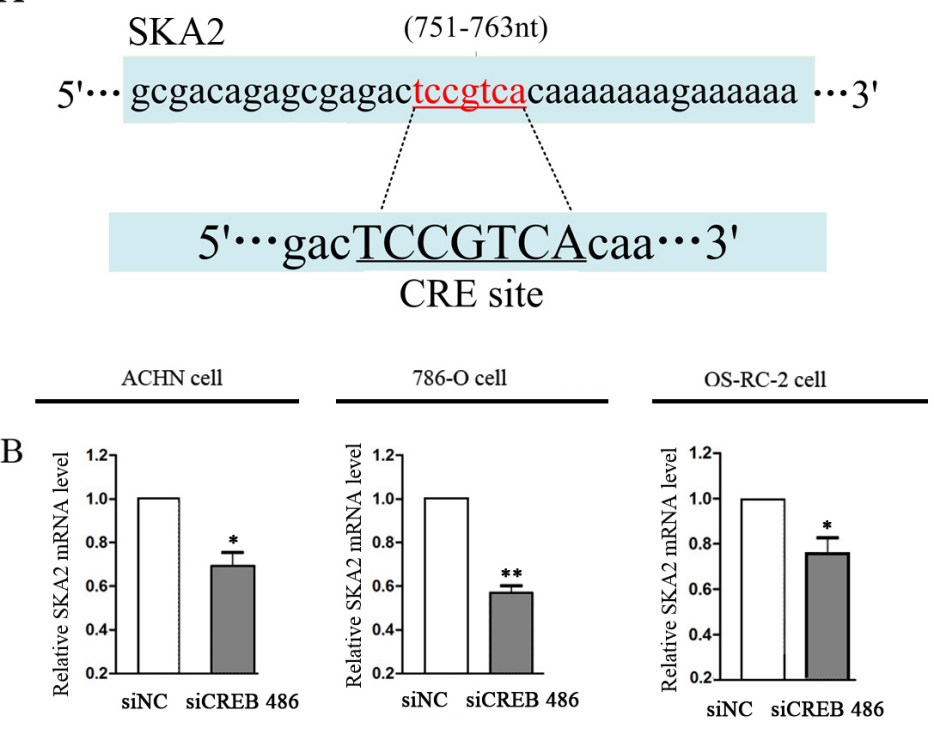

C
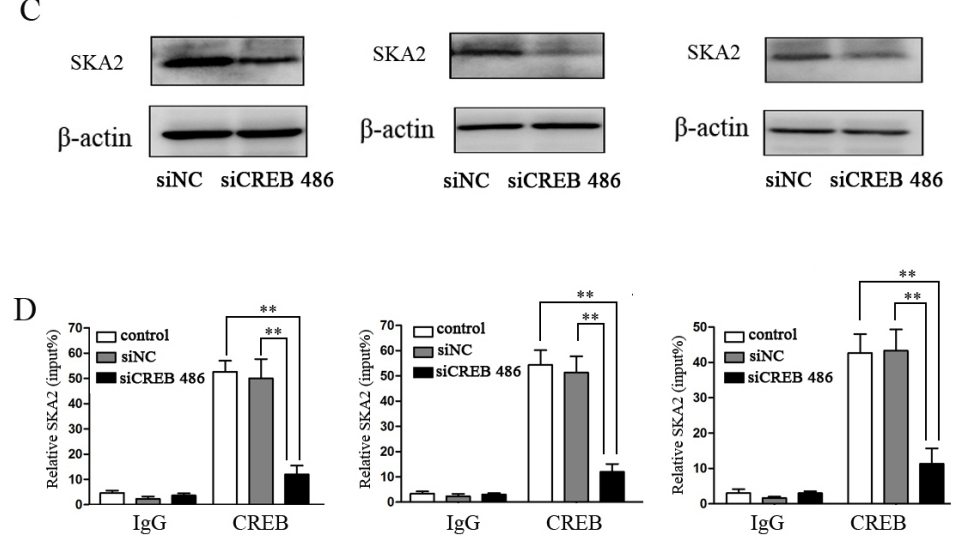

Figure 3: SKA2 is a CREB targeted gene. The potential CREB targeting site in SKA2 promoter, as detected by MultiTF tools (A). After down-regulation of CREB with siCREB, the mRNA (B) and protein (C) levels of SKA2 were significantly reduced in RCC cells. CHIP with specific CREB antibody showed binding of CREB to the putative promoter of SKA2 in RCC cell lines (D). Data were shown as mean $\pm \mathrm{SD}$ from three independent experiments. ${ }^{*} P<0.05, * * P<0.01$ versus siNC treatment. 
A

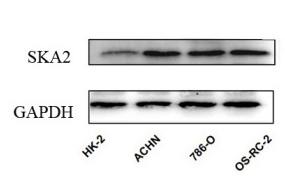

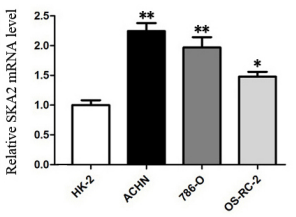
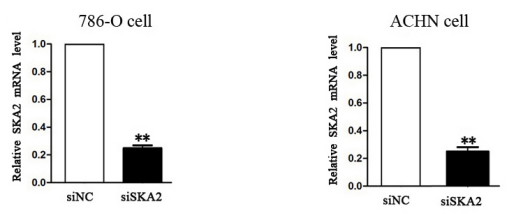

C

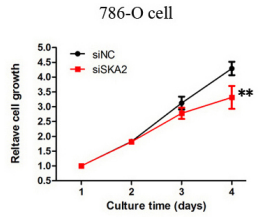

D
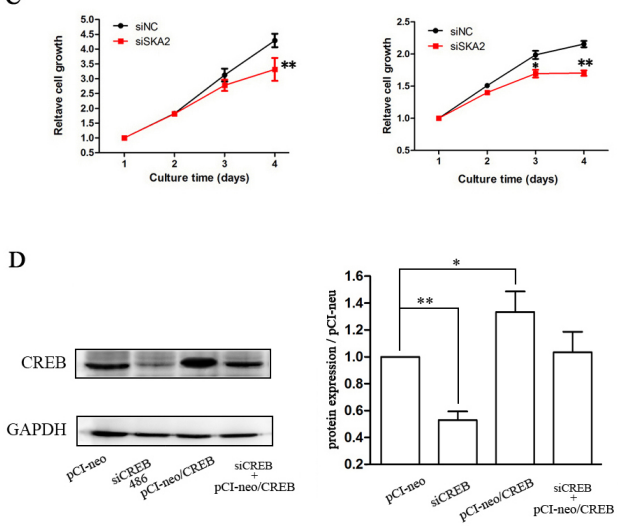

E

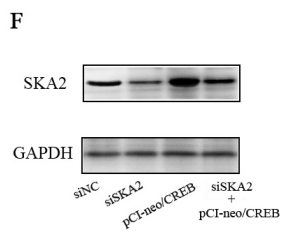

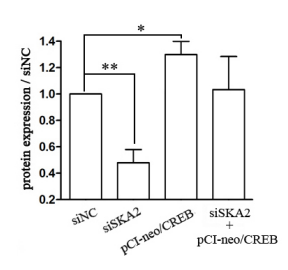

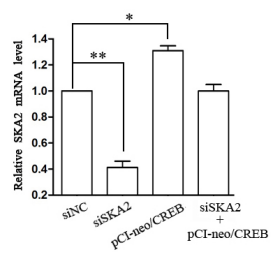

G

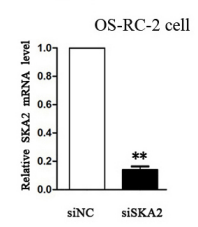

OS-RC-2 cell
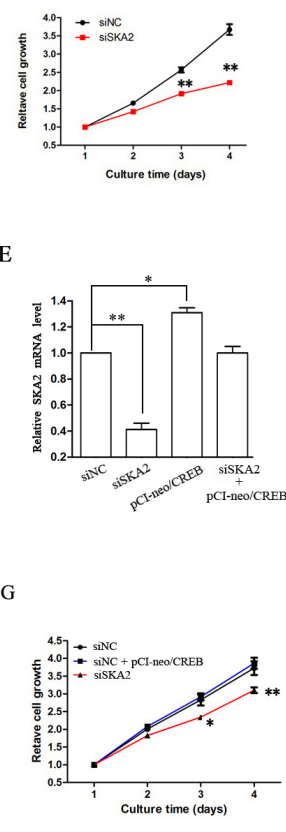

$\mathrm{H}$

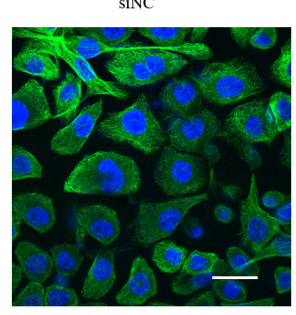

siSKA2

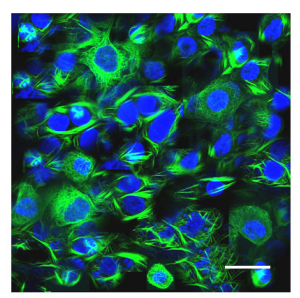

siSKA2 +pCI-neo/CREB

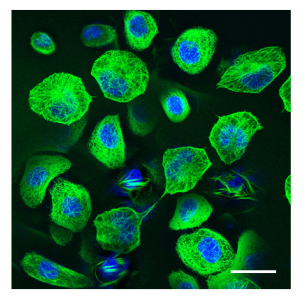

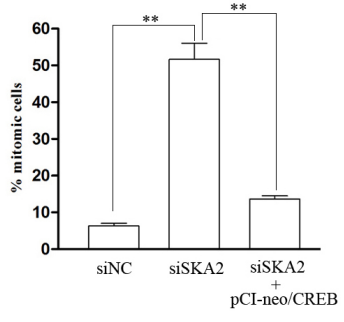

Figure 4: SKA2 is involved in CREB-regulated cell proliferation. The mRNA and protein levels of SKA2 in RCC cells were significantly higher than HK-2 cell (A). After cells were transfected with siSKA2, the mRNA levels were significantly decreased (B), cell proliferation was inhibited (C). However, overexpression of CREB in OS-RC-2 cells co-transfected with pCI-neo/CREB (D), reversed siSKA2-reduced mRNA level $(\mathbf{E})$, protein levels $(\mathbf{F})$, cell growth $(\mathbf{G})$ and cell division $(\mathbf{H})$. Data are shown as mean $\pm \mathrm{SD}$ from three independent experiments. $* P<0.05, * * P<0.01$ versus HK-2 cell or siNC treatment. Scale bar, $10 \mu \mathrm{m}$. 
In order to ascertain the clinical relevance of these findings, we analyzed CREB and SKA2 immunohistochemical staining in RCC specimens $(n=166)$. Intense signals of CREB and SKA2 were observed in the nucleus of tumor cells in TNM stages III and IV RCC tissues, more than those in stages I and II. Very little was seen in the non tumor tissues (Figure 6C). Significant positive correlations were seen between CREB and SKA2 immunostaining in the RCC specimens $(p=0.001, r=0.7)$ (Table 1). These results provided visual corroboration of overexpression of both CREB and SKA2 in RCC tissues, and indicated that CREB
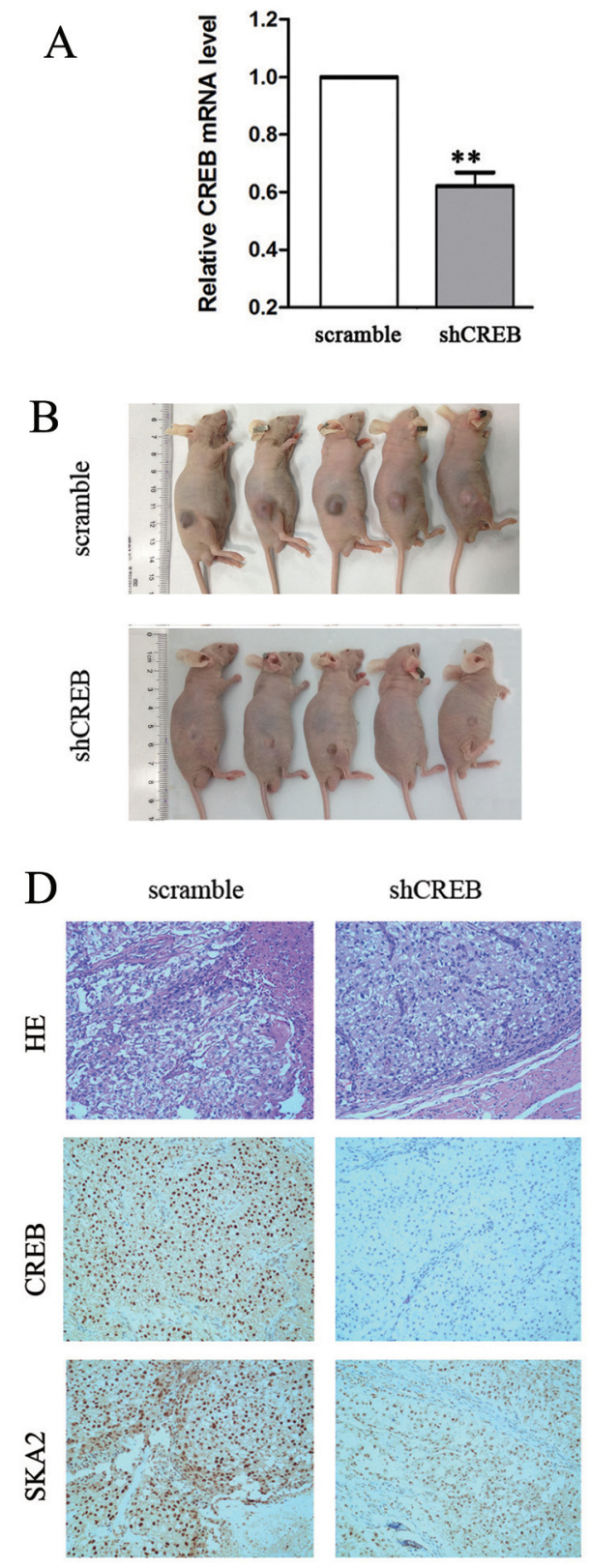

overexpression may be associated with the increase of SKA2 protein levels in RCC at increasing TNM stages.

\section{DISCUSSION}

CREB has been reported to have an impact on carcinogenesis $[1,24]$. In the present study, CREB expression is substantially up-regulated in RCC tissues and cell lines according to the results of Western-blot and qRT-PCR (Figure 1). This is consistent with the results in acute myeloid leukemia [25, 26], glioma [27, 28] and breast cancer [29].

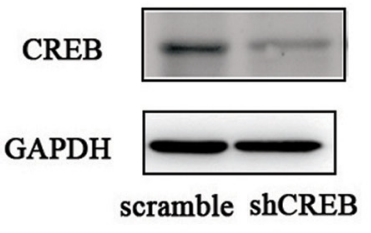

C

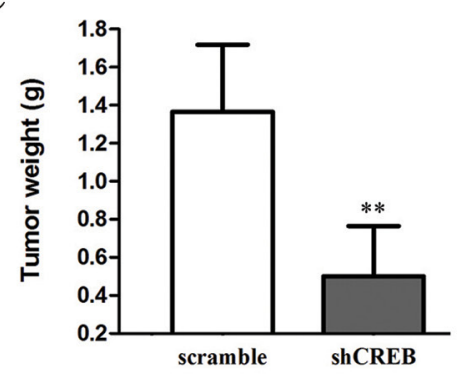

E

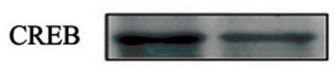

SKA2
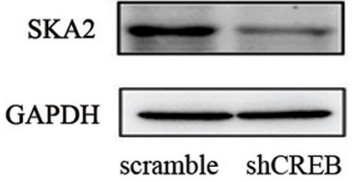

F

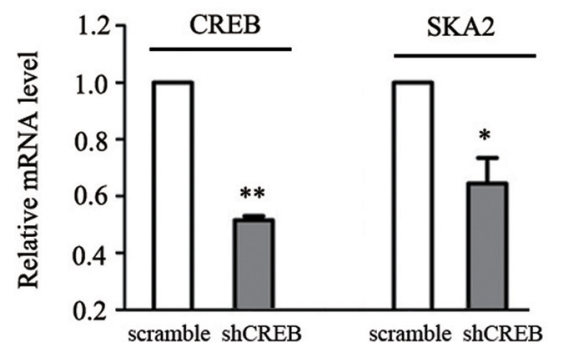

Figure 5: Down-regulation of CREB suppresses xenograft tumor formation and reduces SKA2 expression in vivo. Specific shCREB treatment induced a significant decrease of CREB mRNA and protein levels in OS-RC-2 cells (A). Smaller xenograft tumor formation was shown in shCREB treatment $(\mathbf{B})$, and the average weights of tumors was lower $(\mathbf{C})$ as compared with nude mice injected with OS-RC-2 cells containing scramble. IHC staining showed that the staining score for SKA2 was also lower than in controls (D). Furthermore, the SKA2 protein expression (E) and mRNA level (F) was significantly decreased compared with controls. Data are shown as mean $\pm \mathrm{SD} . * P<0.05, * * P<0.01$ versus scramble. Representative images were captured at a magnification of $\times 200$. 
Table 1: CREB immunohistochemical positive correlates with SKA2 in RCC samples $(n=166)$

\begin{tabular}{cccc}
\hline \multirow{2}{*}{ CREB } & \multicolumn{2}{c}{ SKA2 } & \multirow{2}{*}{ Total } \\
\cline { 2 - 4 } & Low & High & 45 \\
Low & 36 & 9 & 121 \\
High & 11 & 110 & 166 \\
Total & 47 & 119 &
\end{tabular}

$P<0.0001$, Fisher's exact test.

$r=0.7 ; r$, Spearman correlation coefficient.

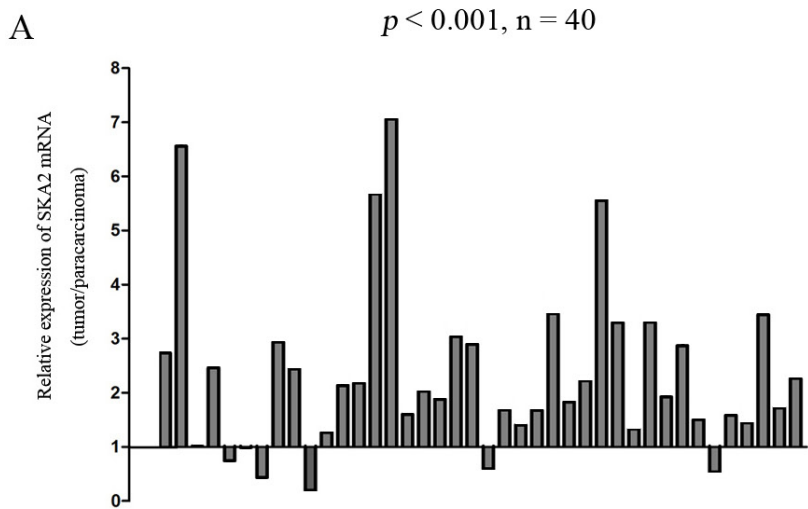

$\mathrm{C}$

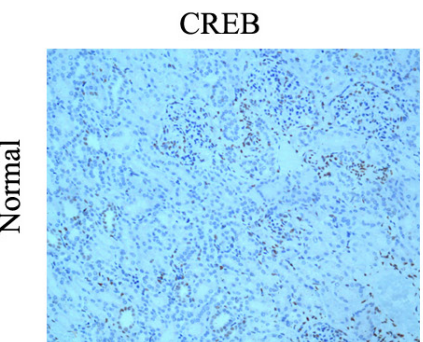

SKA2

B
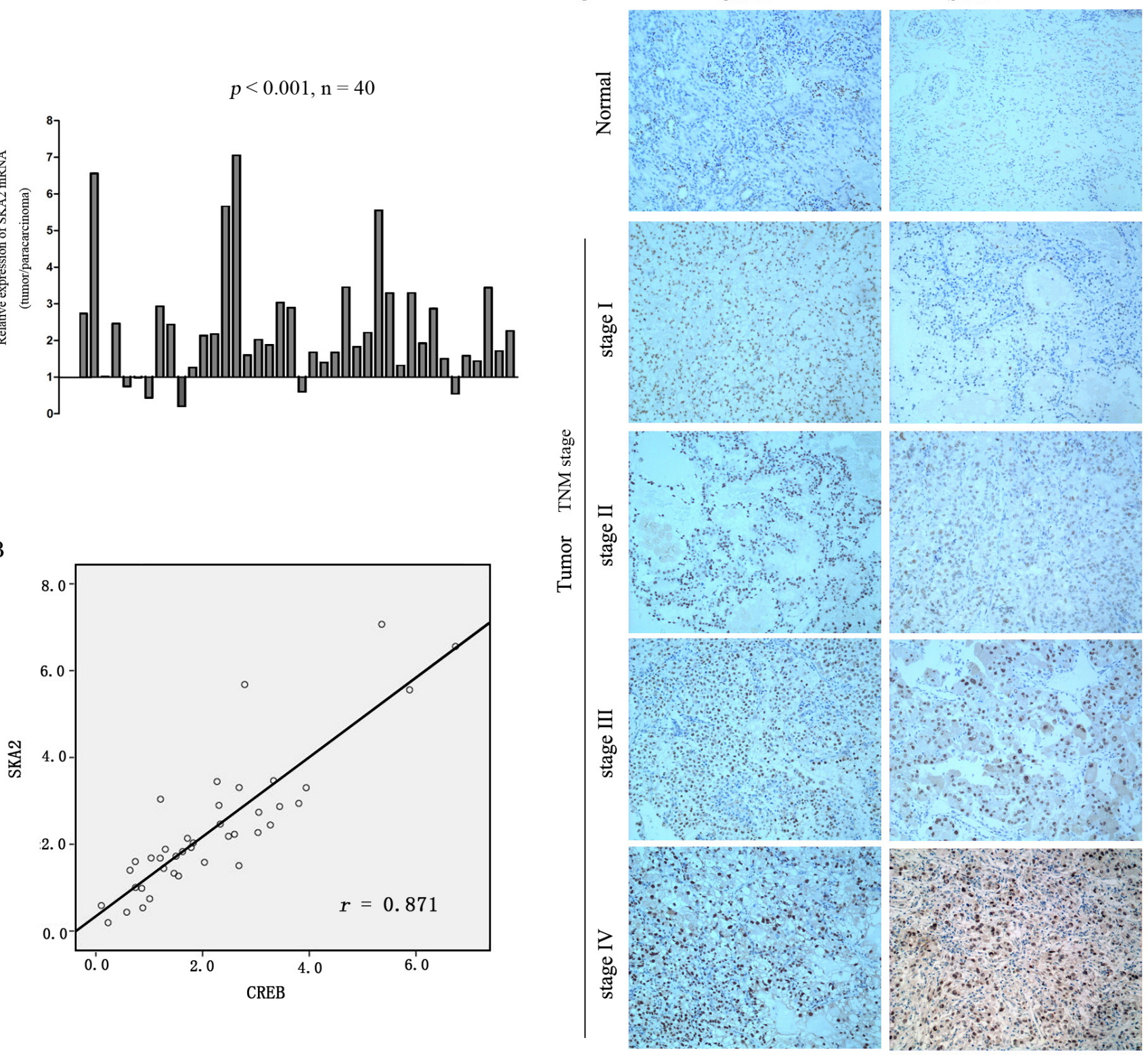

Figure 6: The correlation between SKA2 and CREB expression in RCC tissue sample. The mRNA level of SKA2 was up-regulated in RCC cancers compared to their matched adjacent non-tumor tissues (A). Positive correlation between the mRNA levels of CREB and SKA2 in RCC cancers versus their matched non-tumor counterparts. A statistically significant correlation between CREB expression and SKA2 protein was observed by Pearson's correlation coefficient (B). Representative immunohistochemical staining of higher CREB is concomitant with higher SKA2 in tumor, but not in benign tissues, from the same patient, with increasing expression seen at higher TNM stages from I-IV $(n=166)$ (Figure $\mathbf{C}$ ). Representative images were captured at a magnification of $\times 200$. 
To move on from the concept of CREB expression and increasing proliferation in RCC, we examined targets of CREB using Multi TF tools software (Figure 3A). Having identified SKA2 as a target of CREB, we then discovered the binding of CREB to the promoter sequence of SKA2 using CHIP analysis (Figure 3D). This link has previously been established in lung cancer but not in RCC [30]. Our laboratory and clinical sample studies were then designed to show cause and effect in this developing concept.

We confirmed that CREB expression is increased in ACHN, 786-O and OS-RC-2 RCC cell lines in vitro compared to benign renal tubular HK-2 lines (Figure 1). These data are in line with our previous report regarding pCREB and data from previous studies [22]. In vitro studies using 2 separate transfected siCREB cell lines showed reduced proliferation compared to benign HK-2 controls (Figure 2). We also confirmed that CREB silencing significantly decreased SKA2 expression (Figure 3B, 3C). These studies reinforce the concept in previous reports from our group using pCREB mutation and other publications [2, 22].

SKA2 protein is shown to have a role in regulating progression through mitosis [21], knockdown of SKA2 produced a prolonged checkpoint dependent delay in a metaphase-like state in HeLa and A549 cells [31, 32]. Our Western-blot and qRT-PCR studies of SKA2 levels in $\mathrm{RCC}$ cell lines showed increased expression compared to HK-2. RCC cell line proliferation in vitro was reduced with siSKA2, and this effect was reversed by ectopic administration of CREB, also decreasing the siSKA2 effect of holding numbers of cells in mitosis (Figure 4). To our best knowledge, the in vitro suppression of RCC cell line proliferation by siSKA 2 treatment is shown for the first time by our studies as well as the observation of depleted SKA2 holding RCC cells in mitosis.

Our in vivo data showed that when compared to control, shCREB-treated OS-RC-2 cells achieved reduced tumor growth after 30 days in nude mice. The SKA2 levels in these tumors were reduced in line with reduced tumor growth. This, to our knowledge, is the first report of the association between shCREB and reduced in vivo RCC cell line proliferation accompanied by SKA2 depletion. Our report also seems to be the first to show in vivo link of these effects in any cancer type (Figure 5). Our clinical data shows a strong correlation between nuclear staining of both CREB and SKA2 in RCC cell nuclei. Interesting increases in both CREB and SKA expression were noted in higher TNM stages (Figure 6). From a theoretical basis, knowing the effect of CREB on RCC proliferation, the link with SKA2 is proposed following Genome study. In vitro and in vivo evidence of this link is presented. Corroboration data from clinical samples is provided. Our data support the oncogene effect of CREB mediated by SKA2 in RCC.

While we show convincing data in support of our hypothesis, there are weaknesses in our study as follows: luciferase reporter assays will be helpful to explore the binding CREB to 3'-UTR of SKA2. As yet, we do not have explanations for the clinical RCC samples which did not show up-regulation of CREB. As RCC includes a number of dominant cell types and presumably many genetic mutation and tumorogenesis pathways, the subject is in its infancy and requires vigorous further research. When ectopically administered pCI-neo/CREB appeared to reverse the inhibition of proliferation caused by siSKA2, the mechanism was not fully clear. The in vivo work showing reduction of tumor growth, paralleled with lower SKA2 expression, merits further corroboration with statistically significant data.

\section{CONCLUSIONS}

The present study indicated that CREB functions as tumor oncogene, promoting renal cell cancer proliferation, probably by upgrading SKA2 expression.

\section{MATERIALS AND METHODS}

\section{Tissue samples}

Tumor specimens and adjacent non-tumor renal tissues were collected from the Pathology Department, Ningbo Urology and Nephrology Hospital. All specimens were obtained on the basis of their availability for research purposes and under a protocol approved by the local medical ethics committee of Ningbo Urology and Nephrology Hospital. Written consent was obtained from the patients prior to their participation in the study. The tumors were classified according to the 2009 American Joint Commission for Cancer Staging TNM classification. The clinicopathological characteristics of included patients are summarized in Table 2.

\section{Cell culture and transfections}

Human renal cell carcinoma (RCC) cell lines (786-O, ACHN, OS-RC-2), an immortalized proximal tubule epithelial cell line (HK-2), and HEK-293T were purchased from American Type Culture Collection (ATCC, Shanghai, China). The 786-O and OS-RC-2 were cultured in RPMI 1640 medium (HyClone, UT, USA), ACHN, HK-2 and HEK-293T were cultured in DMEM (HyClone, UT, USA) medium, and supplemented with $10 \%$ fetal bovine serum (FBS, ExCell Bio, Shanghai, China). All cell cultures were carried out in a humidified chamber at $37^{\circ} \mathrm{C}$ with an atmosphere of $5 \% \mathrm{CO}_{2}$.

SiRNA for CREB: siCREB (sence: $5^{\prime}$-GUCUCCAC AAGUCCAAACATT- 3', antisence: 5' -UGUUUGGACU UGUGGAGACTT- 3'), siRNA for SKA2: siSKA2 (sence: 5' -GGCUGGAAUAUGAAAUCAATT- 3', antisence: $5^{\prime}$-UUGAUUUCAUAUUCCAGCCTT- 3') and siRNA for negative control: siNC (sence: $5^{\prime}$-UCCUCCGAACGUG 


\begin{tabular}{|c|c|}
\hline Characteristics of tumor tissues & No. of Patients \\
\hline All patients & 166 \\
\hline \multicolumn{2}{|l|}{ Gender } \\
\hline Male & 90 \\
\hline Female & 76 \\
\hline \multicolumn{2}{|l|}{ Age (mean (minimum-maximum)) } \\
\hline Male & $52(30-78)$ \\
\hline Female & $51(38-76)$ \\
\hline \multicolumn{2}{|l|}{ Classificationof the Common Histological Subtypes } \\
\hline Clear Cell Renal Cell Carcinoma & 150 \\
\hline Papillary Renal Cell Carcinoma & 7 \\
\hline Chromophobe Renal Cell Carcinoma & 9 \\
\hline \multicolumn{2}{|l|}{ Differentiation } \\
\hline Well & 45 \\
\hline Moderate & 88 \\
\hline Low & 33 \\
\hline \multicolumn{2}{|l|}{ Invasion depth } \\
\hline $\mathrm{T} 1$ & 68 \\
\hline $\mathrm{T} 2$ & 53 \\
\hline $\mathrm{T} 3$ & 27 \\
\hline $\mathrm{T} 4$ & 18 \\
\hline \multicolumn{2}{|l|}{ Lymph node metastasis } \\
\hline No & 55 \\
\hline N1 & 64 \\
\hline $\mathrm{N} 2$ & 35 \\
\hline N3 & 12 \\
\hline \multicolumn{2}{|l|}{ Distant metastasis } \\
\hline M0 & 142 \\
\hline Ml & 24 \\
\hline \multicolumn{2}{|l|}{ TNM stage } \\
\hline I & 35 \\
\hline II & 50 \\
\hline III & 46 \\
\hline IV & 35 \\
\hline
\end{tabular}

UCACGUTT- 3', antisence: 5' -ACGUGACACGUUC GGAGAATT- 3') were purchased from GenePharma (Shanghai, China). Transfection of siCREB, siSKA2 or siNC were transfected using the Lipofectamine 2000 transfection reagent (Invitrogen, USA) following the manufacturer's instructions [33].

\section{Cell proliferation assay}

Cell proliferation was examined using CellTiter96 ${ }^{\circledR}$ AQueous One Solution Cell Proliferation Assay (Promega, WI, USA). Briefly, cells transfected with siCREB,
siSKA2 or siNC were plated in a 96-well tissue culture plate. Following incubation for 24, 48, 72, and $96 \mathrm{~h}$, CellTiter $96^{\circledR}$ AQueous One Solution was added to each well and the cells were incubated for another $3 \mathrm{~h}$ at $37^{\circ} \mathrm{C}$. The absorbance at $490 \mathrm{~nm}$ was measured using a spectrophotometer [34].

\section{Western blot analysis}

Cells were lysed and protein was harvested with RIPA buffer (solarbio, Beijing, China) and quantified by bicinchoninic acid (BCA) analysis (Beyotime, Shanghai, 
China). The proteins $(50 \mu \mathrm{g})$ were then separated on $12 \%$ SDS/PAGE gel and then transferred onto PVDF membranes (Millipore, Billerica, MA). After 5\% BSA blocking, membranes were incubated with appropriate dilutions of specific primary antibodies overnight at $4{ }^{\circ} \mathrm{C}$ [35]. The following antibodies were used: anti-CREB, GAPDH (Cell Signaling Technology, MA, USA) and anti-SKA2 antibody (Abcam, Cambridge, UK). Then, membranes were incubated with horseradish peroxidaselabeled secondary antibody (Boster, Wuhan, China). The protein bands were visualized using enhanced chemiluminescence reagent.

\section{DNA, RNA preparation, reverse transcription, gene copy number and quantitative real-time RT-PCR (qRT-PCR) analysis}

Genomic DNA from the RCC cells were extracted using the Tissue DNA kit(Omega, Norcross, GA, USA). Total RNA was isolated from cells using the Trizol reagent (Invitrogen, USA). The amounts of total RNA were quantified using spectrophotometric measurements. cDNA was prepared using a reverse transcription kit (Thermo, USA). Quantitative realtime PCR (qRT-PCR) was performed in duplicate with the fluorescent DNA binding dye SYBR green (Roche, US) to detect the gene copy number and mRNA expression with special primers (Table 3) [36].

\section{Chromatin immunoprecipitation (ChIP)}

For ChIP assay, approximately $1 \times 10^{7}$ cells were harvested in medium and fixed with $1 \%$ formaldehyde. Glycine solution was added at a final concentration of $0.125 \mathrm{M}$ to quench unreacted formaldehyde. Fixed cells were collected by spinning at $1500 \mathrm{rpm}$ for $5 \mathrm{~min}$. ChIP assays were performed using a ChIP Assay Kit (Cell Signaling Technology, Inc, USA) according to the manufacturer's instructions. IgG antibody was from the ChIP Assay Kit. CREB antibody was obtained from (Cell Signaling Technology, Inc, USA). Quantification of immunoprecipitated DNA was performed using qRT-PCR with LightCycler 480 SYBR Green I Master (Roche, US). The primers shown in Table 3 were designed according to the promoter region of SKA2 gene. Values derived from three independent experiments were normalized by background signals (IgG) and presented as percentage of Input chromatin (\% Input) $[37,38]$.

\section{Xenograft model in nude mice}

Five-week-old male nude mice (BALB/C) (Shanghai laboratory animal center, China) were used for xenograft model with a protocol approved by the Institutional Animal Ethics Committee of Ningbo University. $3 \times 10^{6}$ OS-RC-2 cells expressing shCREB or scramble shRNA were injected subcutaneously into the flank of mouse ( $n=5$ for each group). Every effort was made to minimize animal suffering. Thirty days after tumor cells inoculation, the mice were sacrificed. The tumors were weighed, lysed for protein and mRNA preparations (analysed by Western blot and qRT-PCR) and prepared for IHC. The results of IHC were scored in a semiquantitative manner as previously reported [22].

\section{IHC staining and evaluation}

Immunohistochemical (IHC) analysis was performed on formalin-fixed paraffin-embedded tissue sections $(5 \mu \mathrm{m}$ thickness) of RCC tumors both from nude mice model and from clinical samples. After dewaxing and rehydration, sections were treated with $10 \% \mathrm{H}_{2} \mathrm{O}_{2}$. After 5\% BSA blocking, all sections were incubated with the primary antibodies at $4{ }^{\circ} \mathrm{C}$ overnight. The primary antibodies used were: anti-CREB (Cell Signaling Technology, MA, USA) and anti-SKA2 (Abcam, Cambridge, UK) antibodies were diluted at 1:500. The secondary antibody was horseradish peroxidase-goat anti-rabbit IgG (Zhongshan Jinqiao, Beijing, China). Diaminobenzidine was used to develop the signal. Sections were lightly counterstained with hematoxylin [39].

\section{Fluorescent immunocytochemistry}

After cells had grown on poly-L-lysine (PLL)coated coverslips for $24 \mathrm{~h}$, cells were fixed with $4 \%(\mathrm{w} / \mathrm{v})$ paraformaldehyde and $0.5 \%$ Glutaraldehyde in PBS for $5 \mathrm{~min}$, and then permeabilized with $0.1 \%$ Triton $\mathrm{X}-100$ in PBS at room temperature for $15 \mathrm{~min}$. Nonspecific antibody binding was blocked by incubation in blocking buffer, containing $1 \% \mathrm{BSA}$ at room temperature for $60 \mathrm{~min}$. Cells were incubated with $\beta$-tubulin rabbit $\mathrm{mAb}$ conjugated to Alexa Flour 488 (1:500, Cell signaling Technology, MA, USA) in 1\% BSA overnight, DAPI fluorescent dye $1 \mu \mathrm{g} / \mathrm{ml}$, (Sigma, St Louis, MO, USA). Finally, coverslips were washed twice with PBS and photographed using confocal scanning microscopy [40].

\section{Lentiviral vector production}

Lentiviral plasmids containing optimized CREB short hairpin (shRNA) or scramble sequences were produced using $293 \mathrm{~T}$ cells, and were used to infect OSRC-2 cells. The CREB-specific shRNA and scrambled control sequences shRNA were designed using the RNAi Consortium tool (http://www.broadinstitute. org/rnai/trc). The sequences for CREB shRNAs were ACAGCACCCACTAGCACTATT. The sequence for scramble control was CCTAAGGTTAAGTCGCCCTCG. Double-stranded oligonucleotides representing the complementary sequences separated by a hairpin loop were cloned into pLKO.1 puro plasmids. Three plasmids 
Table 3: Primer sequences of CREB and SKA2 for qRT-PCR and ChIP assays

\begin{tabular}{|c|c|}
\hline Gene & Primer sequences \\
\hline CREB & $\begin{array}{l}\text { F: 5'-GAAGATTCACAGGAGTCAGTGGATA-3' } \\
\text { R: 5'-GAAGATTCACAGGAGTCAGTGGATA-3' }\end{array}$ \\
\hline gCREB & $\begin{array}{l}\text { F: 5'-GTCATGGCCTACGAGGAGAA-3' } \\
\text { R: 5'-CACGTCAGGGAGAAGCAGAG-3' }\end{array}$ \\
\hline SKA2 & $\begin{array}{l}\text { FI: 5'-CTGAAACTATGCTAAGTGGGGGAG -3' } \\
\text { R1: 5'-TTCCAAACATCCTGACACTCAAAAG-3' }\end{array}$ \\
\hline SKA2 (for CHIP) & $\begin{array}{l}\text { F2: 5'-GTACACACGCATGCACACAC-3' } \\
\text { R2: 5'-AGGCAAGGGAAATAGGGAAA-3' }\end{array}$ \\
\hline GAPAD & $\begin{array}{l}\text { F: 5'-AAGCCTGCCGGTGACTAAC-3' } \\
\text { R: 5'-GCATCACCCGGAGGAGAAAT-3' }\end{array}$ \\
\hline gGAPAD & $\begin{array}{l}\text { F: 5'-GGTCATCCATGACAACTTTGG-3' } \\
\text { R: 5'-GGCCATCACGCCACAG-3' }\end{array}$ \\
\hline
\end{tabular}

pLKO.1 puro, pCMV-dR8.2 dvpr and pCMV-VSVG were transfected into HEK-293T cells. Lentivirus-containing supernatants were harvested $72 \mathrm{~h}$ after transfection and filtered using $0.45 \mu \mathrm{m}$ cellulose acetate filters, and used to infect OS-RC-2 cells. Subsequently, cells were selected for positive clones using puromycin.

\section{Statistical analysis}

All experiments were repeated three times, except for the mouse cohorts examined in 2 cohorts. The data were expressed as means \pm SD. Statistical analyses were performed with SPSS software. Differences were considered significant if $P<0.05$.

\section{ACKNOWLEDGMENTS}

This work was supported by the grants from National Natural Science Foundation of China (81372209), Scientific Plan of Medical and Health of Zhejiang Province (2015RCB024), Technology foundation of Yinzhou Science and Technology Bureau, China and K. C. Wong Magna Fund in Ningbo University.

\section{CONFLICTS OF INTEREST}

None declared.

\section{REFERENCES}

1. Kinjo K, Sandoval S, Sakamoto KM, Shankar DB. The role of CREB as a proto-oncogene in hematopoiesis. Cell cycle. 2005; 4:1134-1135.

2. Xiao X, Li BX, Mitton B, Ikeda A, Sakamoto KM. Targeting CREB for cancer therapy: friend or foe. Curr cancer drug targets. 2010; 10:384-391.

3. Sakamoto KM, Frank DA. CREB in the pathophysiology of cancer: implications for targeting transcription factors for cancer therapy. Clin Cancer Res. 2009; 15:2583-2587.
4. Zhang X, Odom DT, Koo SH, Conkright MD, Canettieri G, Best J, Chen H, Jenner R, Herbolsheimer E, Jacobsen E, Kadam S, Ecker JR, Emerson B, et al. Genome-wide analysis of cAMP-response element binding protein occupancy, phosphorylation, and target gene activation in human tissues. Proc Natl Acad Sci USA. 2005; 102:4459-4464.

5. Peng B, Lei N, Chai Y, Chan EK, Zhang JY. CIP2A regulates cancer metabolism and CREB phosphorylation in non-small cell lung cancer. Mol Biosyst. 2015; 11:105-114.

6. Seo HS, Liu DD, Bekele BN, Kim MK, Pisters K, Lippman SM, Wistuba II, Koo JS. Cyclic AMP response element-binding protein overexpression: a feature associated with negative prognosis in never smokers with non-small cell lung cancer. Cancer Res. 2008; 68:6065-6073.

7. Liu YL, Lai F, Wilmott JS, Yan XG, Liu XY, Luan Q, Guo ST, Jiang CC, Tseng HY, Scolyer RA, Jin L, ZhangXD. Noxa upregulation by oncogenic activation of MEK/ERK through CREB promotes autophagy in human melanoma cells. Oncotarget. 2014; 5:11237-11251. doi: 10.18632/ oncotarget.2616.

8. Son J, Lee JH, Kim HN, Ha H, Lee ZH. cAMP-responseelement-binding protein positively regulates breast cancer metastasis and subsequent bone destruction. Biochem Biophys Res Commun. 2010; 398:309-314.

9. Singh R, Shankar BS, Sainis KB. TGF-beta1-ROS-ATM-CREB signaling axis in macrophage mediated migration of human breast cancer MCF7 cells. Cell Signal. 2014; 26:1604-1615.

10. Chhabra A, Fernando H, Watkins G, Mansel RE, Jiang WG. Expression of transcription factor CREB1 in human breast cancer and its correlation with prognosis. Oncol Rep. 2007; 18:953-958.

11. Zhang S, Chen L, Cui B, Chuang HY, Yu J, WangRodriguez J, Tang L, Chen G, Basak GW, Kipps TJ. ROR1 is expressed in human breast cancer and associated with enhanced tumor-cell growth. PloS one. 2012; 7:e31127. 
12. Kovach SJ, Price JA, Shaw CM, Theodorakis NG, McKillop IH. Role of cyclic-AMP responsive element binding (CREB) proteins in cell proliferation in a rat model of hepatocellular carcinoma. J Cell Physiol. 2006; 206:411-419.

13. Cho EC, Mitton B, Sakamoto KM. CREB and leukemogenesis. crit Rev Oncog. 2011; 16:37-46.

14. van der Sligte NE, Kampen KR, ter Elst A, Scherpen FJ, Meeuwsen-de Boer TG, Guryev V, van Leeuwen FN, Kornblau SM, de Bont ES. Essential role for cyclic-AMP responsive element binding protein 1 (CREB) in the survival of acute lymphoblastic leukemia. Oncotarget. 2015; 6:14970-14981. doi: 10.18632/oncotarget.3911.

15. Wang P, Yan H, Li JC. CREB-mediated Bcl-2 expression in trichosanthin-induced Hela cell apoptosis. Biochem Biophys Res Commun. 2007; 363:101-105.

16. Wang P, Huang S, Wang F, Ren Y, Hehir M, Wang X, Cai J. Cyclic AMP-response element regulated cell cycle arrests in cancer cells. PloS one. 2013; 8:e65661.

17. Siegel R, Ma J, Zou Z, Jemal A. Cancer statistics, 2014. CA Cancer J Clin. 2014; 64:9-29.

18. Caldwell IR, Oei P, Ng D, Caudwell B, Fong PC, Broom RJ. Analysis of molecular cytogenetic changes in metastatic renal cell carcinoma in the setting of everolimus treatment: a pilot project. Clin Genitourin Cancer. 2014; 12:256-261.

19. Chen Y, Sun Y, Rao Q, Xu H, Li L, Chang C. Androgen receptor (AR) suppresses miRNA-145 to promote renal cell carcinoma (RCC) progression independent of VHL status. Oncotarget. 2015; 6:31203-31215. doi: 10.18632/ oncotarget. 4522 .

20. Ovcharenko I, Loots GG, Giardine BM, Hou M, Ma J, Hardison RC, Stubbs L, Miller W. Mulan: multiple-sequence local alignment and visualization for studying function and evolution. Genome Res. 2005; 15:184-194.

21. Hanisch A, Sillje HH, Nigg EA. Timely anaphase onset requires a novel spindle and kinetochore complex comprising Ska1 and Ska2. EMBO J. 2006; 25:5504-5515.

22. Wang X, Ren Y, Zhuang H, Meng X, Huang S, Li Y, Hehir M, Wang P. Decrease of phosphorylated protooncogene CREB at Ser 133 site inhibits growth and metastatic activity of renal cell cancer. Expert Opin Ther Targets. 2015; 19:985-995.

23. Huang S, Ren Y, Wang P, Li Y, Wang X, Zhuang H, Fang R, Wang Y, Liu N, Hehir M, Zhou JX. Transcription factor CREB is involved in CaSR-mediated cytoskeleton gene expression. Anat Rec (Hoboken). 2015; 298:501-512.

24. Hui K, Yang Y, Shi K, Luo H, Duan J, An J, Wu P, Ci Y, Shi L, Xu C. The p38 MAPK-regulated PKD1/CREB/ Bcl-2 pathway contributes to selenite-induced colorectal cancer cell apoptosis in vitro and in vivo. Cancer Lett. 2014; 354:189-199.

25. Shankar DB, Cheng JC, Kinjo K, Federman N, Moore TB, Gill A, Rao NP, Landaw EM, Sakamoto KM. The role of CREB as a proto-oncogene in hematopoiesis and in acute myeloid leukemia. Cancer cell. 2005; 7:351-362.
26. Cheng JC, Esparza S, Sandoval S, Shankar D, Fu C, Sakamoto KM. Potential role of CREB as a prognostic marker in acute myeloid leukemia. Future Oncol. 2007; $3: 475-480$.

27. Daniel P, Filiz G, Brown DV, Hollande F, Gonzales M, D'Abaco G, Papalexis N, Phillips WA, Malaterre J, Ramsay RG, Mantamadiotis T. Selective CREB-dependent cyclin expression mediated by the PI3K and MAPK pathways supports glioma cell proliferation. Oncogenesis. 2014; 3:e108.

28. Tan X, Wang S, Yang B, Zhu L, Yin B, Chao T, Zhao J, Yuan J, Qiang B, Peng X. The CREB-miR-9 negative feedback minicircuitry coordinates the migration and proliferation of glioma cells. PloS one. 2012; 7:e49570.

29. Detry C, Lamour V, Castronovo V, Bellahcene A. CREB-1 and AP-1 transcription factors JunD and Fra-2 regulate bone sialoprotein gene expression in human breast cancer cells. Bone. 2008; 42:422-431.

30. Cao G, Huang B, Liu Z, Zhang J, Xu H, Xia W, Li J, Li S, Chen L, Ding H, Zhao Q, Fan M, Shen B, et al. Intronic miR-301 feedback regulates its host gene, ska2, in A549 cells by targeting MEOX2 to affect ERK/CREB pathways. Biochem Biophys Res Commun. 2010; 396:978-982.

31. Rice L, Waters CE, Eccles J, Garside H, Sommer P, Kay P, Blackhall FH, Zeef L, Telfer B, Stratford I, Clarke R, Singh D, Stevens A, et al. Identification and functional analysis of SKA2 interaction with the glucocorticoid receptor. J Endocrinol. 2008; 198:499-509.

32. Vigneron S, Brioudes E, Burgess A, Labbe JC, Lorca T, Castro A. RSK2 is a kinetochore-associated protein that participates in the spindle assembly checkpoint. Oncogene. 2010; 29:3566-3574.

33. Hunt MA, Currie MJ, Robinson BA, Dachs GU. Optimizing transfection of primary human umbilical vein endothelial cells using commercially available chemical transfection reagents. J Biomol Tech. 2010; 21:66-72.

34. Jiang L, Xu Y, Zeng X, Fang J, Morse IH, Zhou JX. Suppression of CD300A inhibits the growth of diffuse large B-cell lymphoma. Oncotarget. 2015; 6:31191-31202. doi: 10.18632/oncotarget.5152.

35. Wang $\mathrm{P}, \mathrm{Xu} \mathrm{J}$, Zhang C. CREB, a possible upstream regulator of Bcl-2 in trichosanthin-induced HeLa cell apoptosis. Mol Biol Rep. 2010; 37:1891-1896.

36. Dagan LN, Jiang X, Bhatt S, Cubedo E, Rajewsky K, Lossos IS. miR-155 regulates HGAL expression and increases lymphoma cell motility. Blood. 2012; 119:513-520.

37. Lesiak A, Pelz C, Ando H, Zhu M, Davare M, Lambert TJ, Hansen KF, Obrietan K, Appleyard SM, Impey S, Wayman GA. A genome-wide screen of CREB occupancy identifies the RhoA inhibitors Par6C and Rnd3 as regulators of BDNF-induced synaptogenesis. PloS one. 2013; 8:e64658.

38. Han L, Zhang EB, Yin DD, Kong R, Xu TP, Chen WM, Xia R, Shu YQ, De W. Low expression of long noncoding 
RNA PANDAR predicts a poor prognosis of non-small cell lung cancer and affects cell apoptosis by regulating Bcl-2. Cell Death Dis. 2015; 6:e1665.

39. Xu Y, Jiang L, Fang J, Fang R, Morse HC, 3rd, Ouyang G, Zhou JX. Loss of IRF8 Inhibits the Growth of Diffuse Large B-cell Lymphoma. J Cancer. 2015; 6:953-961.
40. Wang P, Li JC. Trichosanthin-induced specific changes of cytoskeleton configuration were associated with the decreased expression level of actin and tubulin genes in apoptotic Hela cells. Life Sci. 2007; 81:1130-1140. 